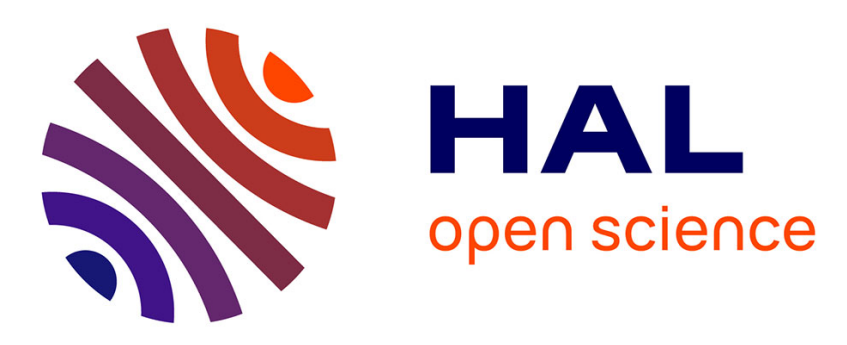

\title{
Sustainable Series of New Epoxidized Vegetable Oil-Based Thermosets with Chemical Recycling Properties
}

Chiara Di Mauro, Samuel Malburet, Aratz Genua, Alain Graillot, Alice Mija

\section{- To cite this version:}

Chiara Di Mauro, Samuel Malburet, Aratz Genua, Alain Graillot, Alice Mija. Sustainable Series of New Epoxidized Vegetable Oil-Based Thermosets with Chemical Recycling Properties. Biomacromolecules, 2020, 21 (9), pp.3923 - 3935. 10.1021/acs.biomac.0c01059 . hal-03007302

\section{HAL Id: hal-03007302 \\ https://hal.univ-cotedazur.fr/hal-03007302}

Submitted on 27 Jan 2021

HAL is a multi-disciplinary open access archive for the deposit and dissemination of scientific research documents, whether they are published or not. The documents may come from teaching and research institutions in France or abroad, or from public or private research centers.
L'archive ouverte pluridisciplinaire HAL, est destinée au dépôt et à la diffusion de documents scientifiques de niveau recherche, publiés ou non, émanant des établissements d'enseignement et de recherche français ou étrangers, des laboratoires publics ou privés. 


\title{
Sustainable Series of New Epoxidized Vegetable Oil-Based Thermosets with Chemical Recycling Properties
}

\author{
Chiara Di Mauro, Samuel Malburet, Aratz Genua, Alain Graillot, and Alice Mija* \\ Cite This: Biomacromolecules 2020, 21, 3923-3935 \\ Read Online
}

ABSTRACT: This work reports for the first time the copolymerization studies of 11 newly synthesized epoxidized vegetable oils (EVOs) that reacted with a disulfide-based aromatic dicarboxylic acid (DCA) to produce thermoset materials with recyclability properties. These new EVOs' reactivity and properties were compared with those of the two commercial references: epoxidized linseed oil (ELO) and epoxidized soybean oil (ESO). The structure-reactivity correlation is proposed by differential scanning calorimetry (DSC) analysis, corroborating the epoxy content of EVO monomers, the initiator effect, the copolymerization reaction enthalpy, and the temperature range. The thermomechanical properties of the obtained

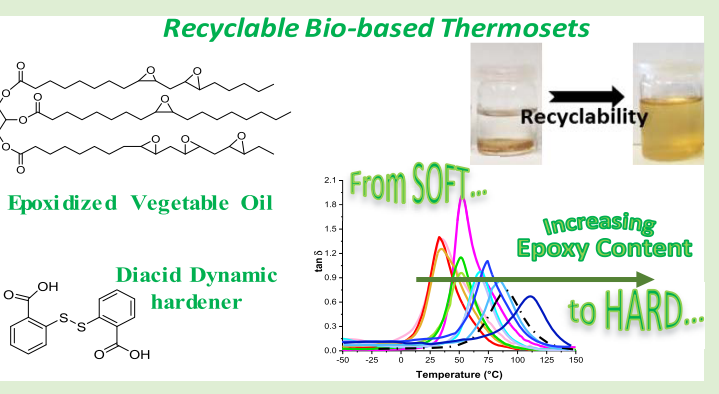
thermosets were evaluated and discussed in correlation with the structure and reactivity of monomers by dynamic mechanical analysis (DMA), tensile testing, and thermogravimetric analysis (TGA). It has been found that the higher the EVO functionality, the higher is the reactivity, cross-linking density, and final performances, with $\tan \delta$ values ranging from 34 to $111{ }^{\circ} \mathrm{C}$. This study investigates the chemical recycling and the solvent resistance of these vitrimerlike materials that have a high bio-based carbon content, from 58 to $79 \%$, with potential application in coating or composite materials in the automotive sector.

\section{INTRODUCTION}

Accumulation of plastic waste is becoming one of the major threats to our ecosystem. With a level of production of around 140 million tons of synthetic polymers each year and with less than $10 \%$ being effectively recycled, the growing amount of plastic waste ending up in oceans or landfills raises several concerns. Developed countries, as the main consumers of energy, for a long time, started planning and managing new renewable and alternative energy sources to solve the current problems related to air quality linked to ozone, carbon monoxide, or particulate matter. The sustainable use of natural resources is one of the strategies to ensure the development of renewable materials and a sustainable economy. In the last few decades, the use of bio-based raw materials has led to the development of green chemistry by integrating minor toxicity ${ }^{1}$ and biodegradability, ${ }^{2-4}$ offering a different scenario for coal as a basic feedstock. ${ }^{5}$ However, most of these products still do not offer a competitive alternative compared with the products coming from the petrochemical industries. In the case of thermoset materials, this problem is further duplicated since they cannot be recycled. Although relentless efforts are dedicated to reduce the use of petro-based resources, the ecological impact of thermosets at the end of the lifetime remains a crucial challenge that needs to be addressed to limit the greenhouse emissions and the associated footprint. For this reason, important strategies are the replacing of petro-based raw materials and also the synthesis of recyclable, reusable, and reshapable vitrimer-like thermoset materials based on rever- sible disulfide bond and carboxylate transesterification (vitrimers). ${ }^{6-8}$

Considering the growing amount of fiber-reinforced composite materials due to their outstanding properties in terms of lightweight, high weight-to-strength ratio, and long durability, the next generation of bio-based thermosetting resins need to be cost-effective and based on nondepletable bio-based resources. Within this scope, the valorization of vegetable oils through the synthesis of thermoset materials is supported by advantages like diversity, abundance, and low price, promising to be a sustainable alternative for the chemical industry. 9

Commonly, vegetable oils (VOs) are extracted from fruits or seeds by solvent extraction or by the pressing process. More than $95 \%$ of VOs constitute triglycerides, ${ }^{10}$ containing three fatty acids branched to a glycerol unit. Nevertheless, VOs are a complex, variable, and thus very interesting mixture. The diversity in VO composition depends not only on the nature of the oil but also on the geographical origin of the plants, maturity, species, and the method used for its extraction. As a

Received: July 14, 2020

Revised: August 10, 2020

Published: August 13, 2020 
Table 1. Chemical Composition of VO Raw Materials and Corresponding EVOs

\begin{tabular}{|c|c|c|c|c|c|}
\hline \multirow[b]{2}{*}{ EVOs } & \multicolumn{4}{|c|}{ fatty acid } & \multirow[b]{2}{*}{ epoxy index (mequiv $\cdot \mathrm{g}^{-1}$ ) } \\
\hline & saturated $^{e}$ & monounsaturated $^{f}$ & diunsaturated $^{g}$ & triunsaturated $^{h}$ & \\
\hline karanja $^{a}$ & 22 & 56 & 21 & 1 & 2.77 \\
\hline castor $^{a}$ & 3 & $92^{d}$ & 5 & & 2.85 \\
\hline St John's wort ${ }^{a}$ & 21 & 63 & 16 & & 2.97 \\
\hline peanut $^{b}$ & 20 & 53 & 27 & & 3.35 \\
\hline rapeseed $^{a}$ & 7 & 62 & 25 & 7 & 3.99 \\
\hline soybean & 11 & 24 & 54 & 8 & 4.20 \\
\hline rosehip seed ${ }^{a}$ & 17 & 20 & 57 & 5 & 4.7 \\
\hline safflower $^{c}$ & 12 & 18 & 70 & & 4.93 \\
\hline grapeseed $^{b}$ & 11 & 22 & 67 & & 4.94 \\
\hline camelina $^{c}$ & 10 & 30 & 28 & 32 & 5.29 \\
\hline linseed & 6 & 22 & 16 & 52 & 5.61 \\
\hline hemp $^{c}$ & 10 & 12 & 65 & 13 & 6.09 \\
\hline perilla $^{c}$ & 3 & 21 & 18 & 58 & 6.77 \\
\hline
\end{tabular}

${ }^{a}$ Nonedible oils. Rapeseed oil is the high erucic fatty acid compared to canola, which has low erucic fatty acid. ${ }^{b}$ Edible oils. ${ }^{c}$ Edible but nonvaluated. ${ }^{d}$ Monounsaturated fatty acids of castor oil comprised $90 \%$ of ricinoleic acid. ${ }^{e}$ Mainly comprised of palmitic and stearic fatty acids. ${ }^{f_{M}}$ Mainly comprised of oleic fatty acid. ${ }^{g}$ Mostly linoleic fatty acid. ${ }^{h}$ Mostly linolenic fatty acid.

matter of fact, the length of the main fatty acids is in the range of 4-28 carbons and unsaturated bonds or functional groups such as hydroxyl (castor oil) or epoxy (vernonia oil) can be encountered alongside the alkyl chain. ${ }^{11}$ According to the unsaturation degree of fatty acids, they can be generally classified into saturated, mono- and polyunsaturated fatty acids, which may have different configurations, resulting in different physical and chemical properties. For instance, vegetable oils can be either liquid or waxy at room temperature. This property is mainly linked to two major criteria, which can help to understand the composition of the oil. First, the vegetable oil is certainly poor in unsaturated fatty acids, which allow them to crystallize by self-organization, as in the case of coconut oil or palm oil, for instance. Also, the fatty acids having a long alkyl chain length will promote selforganization and crystallization. In fact, the higher the melting point of the vegetable oil, the higher is its content in saturated fatty acids with long alkyl chains. Finally, the unsaturation degree, also known as the iodine value, has a major importance for the production of monomers and thermosetting resins. High iodine values indicate a high aptitude for functionalization and polymerization. ${ }^{12}$

Usually, the industrial process to synthesize the commercial epoxy resins is based on epichlorohydrin as the main reactant, allowing the conversion of hydroxyl, ${ }^{13}$ phenol, ${ }^{14}$ or acid ${ }^{15}$ functions into epoxides. Vegetable oils have been used for long time as paints, lubricants, ${ }^{16}$ and coatings, but in the last few decades, polymeric systems based on these raw materials have been developed using a variety of polymerization techniques. ${ }^{11}$ One considerable advantage of the use of VOs as raw materials is their potential of double-bond functionalization, as for example, into epoxies using oxidizing agents and, thus, avoiding the very hazardous epichlorohydrin, the usual reactant for diglycidyl ether of bisphenol A (DGEBA) synthesis.

Therefore, based on a wide variety of VOs, we were able to develop and implement a robust, sustainable, and harmless synthetic pathway giving access to fully bio-based epoxidized vegetable oils exhibiting a wide range of epoxy content from 2.77 to 6.77 mequiv $\cdot g^{-1}$. The details and the methodology implemented for new epoxidized vegetable oil (EVO) synthesis are reported elsewhere. ${ }^{17,18}$ It has been then important to evaluate the potential of synthesized EVOs as precursors for thermoset materials and to highlight how the thermomechanical properties of the obtained materials can be influenced by their epoxy content. The latter is determined by the relative proportion of palmitic (saturated), stearic (saturated), oleic (monounsaturated), linoleic (diunsaturated), and linolenic (triunsaturated) fatty acids in the composition of the corresponding VOs (Table 1). Moreover, considering the rising demand for vegetable oils, notably due to the population growth, a careful selection of vegetable oil feedstocks has been carried out to avoid food competition.

For the first time in a preliminary work, we showed the recycling properties of epoxidized linseed oil (ELO)-based thermosets. ${ }^{19-21}$ In the present work, we developed a new series of vegetable-based thermosetting resins, by the copolymerization of 12 newly synthesized EVOs with 2,2'dithiodibenzoic acid (DTBA). Through this study, the 12 new EVOs were synthesized with the objective to cover the larger domain of the epoxy content, i.e., from 2.77 to 6.77 mequiv. $\mathrm{g}^{-1}$. In this manner, we will gain knowledge on the potential of EVOs to generate thermosets when reacted with DTBA and on their properties. To our knowledge, this is the first study proposing the cross-linking of EVO series with DTBA. If the literature is rich in reporting studies dedicated to EVO crosslinking with anhydrides or aliphatic diacids, there are no much studies on EVO curing using aromatic diacids, and with aromatic diacids containing disulfide linkage are not at all present. Indeed, these $S-S$ linkages allow the incorporation of dynamic cross-links to form covalent adaptable networks $(\mathrm{CANs})^{22}$ that conduct to thermosets with reprocessable, recyclable, and reshapable abilities. Disulfide dynamic bonds have attracted much attention in the design of a series of CANs, as displayed using 4-aminophenyl disulfide (AFD) as the cross-linker for DGEBA by Odriozola et al. ${ }^{23}$ or for the castor oil (CO)-derived polyurethane by Chen et al. ${ }^{24}$ To our knowledge, this paper investigates for the first time how the epoxy content characterizing the new EVOs impact on the copolymerization reactivity with the DCAs and, therefore, on the performances of the corresponding epoxy/acid vitrimerlike thermosets.

The reactivity study was achieved by differential scanning calorimetry analysis (DSC). The structural evolution of the thermosetting resins was studied by attenuated total reflection- 


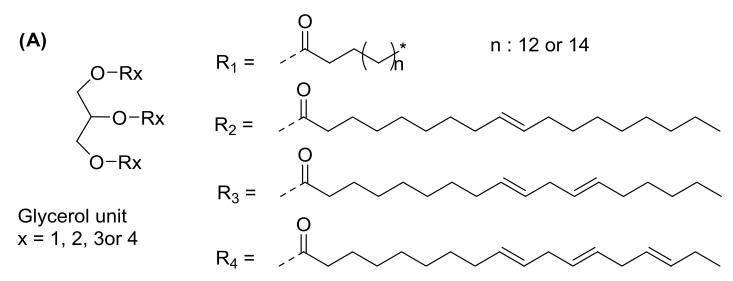

(C)

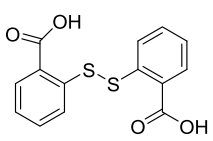

(D)

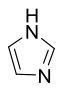

(B)

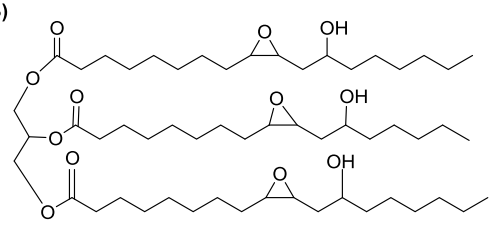

(F)

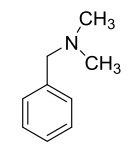

Figure 1. Structures of selected reagents.

Fourier transform infrared spectroscopy (ATR-FTIR). Thereafter, the thermomechanical properties of the thermosets were analyzed by DSC, thermogravimetric analysis (TGA), dynamic mechanical analysis (DMA), and tensile testing. Finally, their solvent stability and network degradability were investigated as a possible option of recycling mechanism. Therefore, this work gives an overview, a complete study, from thermoset synthesis to a detailed investigation of their properties, for a large series of newly epoxidized vegetable oils cross-linked with an aromatic diacid hardener.

\section{MATERIALS AND METHODS}

Materials. The chemical structures of the reagents used in this study are shown in Figure 1. The new biorenewable epoxidized monomers are classified based on the epoxy content as tabulated in Table 1. Figure 1A exhibits the general structure of the EVOs and that of the epoxidized castor oil (ECO) in Figure 1B.

ELO and epoxidized soybean oil (ESO) were generously provided by Valtris Chemicals (England), while the new EVOs (SP-3S-30-005 and SP-3S-30-006) by SPECIFIC POLYMERS (France). Their chemical characteristics are reported in Table S1.

The hardener and the initiators are commercially available and were purchased from Sigma-Aldrich and used as received without further purification: 2,2'-dithiodibenzoic acid (DTBA, 95\%) (C), imidazole (IM, 99\%) (D), 1-methylimidazole (1-MI, 99\%) (E), and $\mathrm{N}, \mathrm{N}$-dimethylbenzylamine (DMBA, 99\%) (F).

Sample Preparation. The thermosetting formulations were carried out by mixing the EVO monomer with the DTBA at a $1: 1$ molar ratio between the epoxy and acid groups, according to the method by Dusek et al. ${ }^{21,25}$ The selected initiator was added at $1 \mathrm{wt} \%$ and mixed at $80{ }^{\circ} \mathrm{C}$ with the previous mixture. The curing and postcuring protocols for the preparation of the EVO thermosets were determined based on the DSC study of reactivity presented in Figure S2 and Table S3. The DSC analysis allowed one to determine for each formulation the optimal temperatures of curing and postcuring, i.e., those temperatures conducting to a complete reaction (no residual enthalpy in the second scan during heating from 25 to $250{ }^{\circ} \mathrm{C}$ at 10 ${ }^{\circ} \mathrm{C} \cdot \mathrm{min}^{-1}$ ) and to the highest $T_{\mathrm{g}}$ values of the obtained thermosets.

Characterization Techniques. Differential Scanning Calorimetry (DSC). The copolymerization reactions were carried out on a Mettler Toledo DSC 3 instrument equipped with STAR software from 25 to $220{ }^{\circ} \mathrm{C}$. The samples (about $10-15 \mathrm{mg}$ ) were placed in $100 \mu \mathrm{L}$ aluminum crucibles. The temperature rate for all scanning runs was at $10{ }^{\circ} \mathrm{C} \cdot \mathrm{min}^{-1}$. The DSC technique was also used to determine the thermosets' glass transition using the same heating rate, over a temperature range from -80 to $180^{\circ} \mathrm{C}$.

Fourier Transform Infrared Spectroscopy (FTIR). FTIR analyses were performed using a Thermo Scientific Nicolet iS50 FTIR spectrometer with a deuterated L-aniline-doped triglycine sulfate (DLaTGS) detector in an attenuated total reflectance (ATR) mode. The absorption bands were recorded in the range of $4000-525 \mathrm{~cm}^{-1}$ at $4 \mathrm{~cm}^{-1}$ resolution and 32 scans. The data were analyzed using OMNIC software. The percentage of the functional group conversion is defined using eq 1

$$
\%=\frac{\left(\frac{A 823}{A 1586}\right)_{0}-\left(\frac{A 823}{A 1586}\right)_{t}}{\left(\frac{A 823}{A 1586}\right)_{0}} \times 100
$$

where area absorbance peaks were calculated at the initial time $\left(A_{0}\right)$ and at the end of the curing protocol $\left(A_{\mathrm{t}}\right)$. The peak at $823 \mathrm{~cm}^{-1}$ corresponds to oxirane $\mathrm{C}-\mathrm{O}$ groups and that at $1586 \mathrm{~cm}^{-1}$ is the reference band, which belongs to the $\delta_{\mathrm{C}-\mathrm{C}}$ of the aromatic signal.

Thermogravimetric Analysis (TGA). TGA measurements were carried out on a Mettler Toledo TGA 2. The microbalance has a precision of $\pm 0.1 \mu \mathrm{g}$. The samples of about $10 \mathrm{mg}$ were placed in 70 $\mu \mathrm{L}$ alumina pans. To characterize the thermal stability of the thermosets, the samples were heated at $10{ }^{\circ} \mathrm{C} \cdot \mathrm{min}^{-1}$ from 25 to 1000 ${ }^{\circ} \mathrm{C}$ under $50 \mathrm{~mL} \cdot \mathrm{min}^{-1}$ air flow.

The statistic heat-resistant index temperature $\left(T_{s}\right)$ is a characteristic of the thermal stability and was calculated according to eq 2

$$
T_{s}=0.49 \times\left[T_{5 \%}+0.6 \times\left(T_{30 \%}-T_{5 \%}\right)\right]
$$

where $T_{5 \%}$ and $T_{30 \%}$ are the temperatures at, respectively, 5 and $30 \%$ weight loss.

Dynamic Mechanical Analysis (DMA). DMA analyses were carried out on a Mettler Toledo DMA 1 instrument, equipped with STAR ${ }^{\odot}$ software for curve analysis. The analyzed samples were prepared according to the curing protocol given in Table S3 and had rectangular dimensions of $30 \times 7 \times 2 \mathrm{~mm}^{3}$ (length $\times$ width $\times$ thickness). Elastic modulus values $\left(E^{\prime}\right)$ and damping factors $(\tan \delta)$ were collected at $3{ }^{\circ} \mathrm{C} \cdot \mathrm{min}^{-1}$ heating rate from -50 to $170{ }^{\circ} \mathrm{C}$ and 1.0 $\mathrm{Hz}$ frequency. The DMA was operated using the tension method. The glass transition was assigned at the maximum of the damping factor $\left(\tan \delta=E^{\prime \prime} / E^{\prime}\right)$. The cross-linking density of EVO thermosets was calculated using eq 3, according to Flory theory of the rubber elasticity $^{26}$

$$
\nu=\frac{E^{\prime}}{3 R T}
$$

where $E^{\prime}$ is the storage modulus in the rubbery plateau region at $T_{\mathrm{g}}+$ $50{ }^{\circ} \mathrm{C}, R$ is the gas constant, and $T$ is the absolute temperature in Kelvin.

Tensile Testing. The tensile tests were performed on a mechanical universal testing machine Instron, model 3365, controlled by BlueHill Lite software developed by Instron (Norwood, MA), according to standard ISO 527-141 and ASTM D638-0842.

To produce specimens for tensile tests, the reactive mixture was stirred at $80{ }^{\circ} \mathrm{C}$ for $10 \mathrm{~min}$, placed into a silicone mold, and cured in an oven, according to Table S3 that summarizes the curing and postcuring protocols. These protocols were previously determined, based on DSC studies of reactivity for each formulation. The tensile bars were produced using silicon molds allowing one to obtain 
Table 2. Reaction Enthalpy, Interval, and Temperature of the Reaction Peak $\left(T_{\text {peak }}\right)$ of EVOs/DTBA/IM Systems during the Copolymerization Reaction

\begin{tabular}{|c|c|c|c|c|c|}
\hline VOs feedstock & EVOs in copolymerization systems & epoxy content (mequiv $\cdot \mathrm{g}^{-1}$ ) & $\Delta H\left(\mathrm{~J} \cdot \mathrm{g}^{-1}\right)$ & $T_{\text {peak }}\left({ }^{\circ} \mathrm{C}\right)$ & reaction interval \\
\hline karanja oil & EKRNO & 2.77 & $84 \pm 3$ & $164 \pm 1$ & $145-186$ \\
\hline castor oil & $\mathrm{ECO}$ & 2.85 & $109 \pm 3$ & $147 \pm 1$ & $130-170$ \\
\hline St John's wort oil & ESJWO & 2.97 & $108 \pm 3$ & $168 \pm 1$ & $154-186$ \\
\hline peanut oil & EPO & 3.35 & $112 \pm 3$ & $171 \pm 1$ & $151-192$ \\
\hline rapeseed oil & ERPO & 3.99 & $138 \pm 3$ & $164 \pm 1$ & $147-184$ \\
\hline soybean oil & ESO & 4.20 & $149 \pm 3$ & $159 \pm 1$ & $142-182$ \\
\hline rosehip seed oil & ERHO & 4.7 & $155 \pm 3$ & $161 \pm 1$ & $140-189$ \\
\hline safflower oil & ESFO & 4.93 & $172 \pm 3$ & $158 \pm 1$ & $142-180$ \\
\hline grapeseed oil & EGRO & 4.94 & $175 \pm 3$ & $162 \pm 1$ & $139-182$ \\
\hline camelina oil & ECMO & 5.29 & $188 \pm 3$ & $153 \pm 1$ & $136-176$ \\
\hline linseed oil & ELO & 5.61 & $197 \pm 3$ & $151 \pm 1$ & $131-180$ \\
\hline hemp oil & EHO & 6.09 & $194 \pm 3$ & $160 \pm 1$ & $135-190$ \\
\hline perilla oil & EPLO & 6.77 & $207 \pm 3$ & $152 \pm 1$ & $126-187$ \\
\hline
\end{tabular}

samples of dimensions $75 \times 10 \times 2 \mathrm{~mm}^{3}$ (length $\times$ width $\times$ thickness).

The analyses were performed using a crosshead speed of $10 \mathrm{~mm}$. $\mathrm{min}^{-1}$. For each formulation, five samples were tested to evaluate the average calculations of tensile properties (Young's modulus, tensile strength, and elongation at break).

Density Calculation. Density was calculated by measuring the volume of each sample and its weight using a Mettler Toledo ML $3002 \mathrm{~T}$ precision balance $( \pm 0.0001 \mathrm{~g})$.

Solvent Stability. The stability of thermosets in solvents was studied in methanol, ethanol, acetone, toluene, $1 \mathrm{~N} \mathrm{NaOH}$, and $1 \mathrm{~N}$ $\mathrm{HCl}$ for $72 \mathrm{~h}$ at room temperature to confirm the complete crosslinking and to evaluate the sample resistance in these solvents. Toluene was chosen as a good solvent for the swelling test, the swelling factor $\left(Q_{\mathrm{m}}\right)$ being calculated according to eq 6 . The materials' cross-link density was determined using Flory-Rehner theory ${ }^{26,27}$ according to eq 4

$$
\nu=\frac{\ln \left(1-\nu_{2, s}\right)+\nu_{2, s}+X_{1} \nu_{2, s}^{2}}{\nu_{1}\left(\frac{\nu_{2, s}}{2}-\sqrt[1 / 3]{\nu_{2, s}}\right)}
$$

where $\nu_{1}$ is the molar volume of the solvent (for toluene $\nu_{1}=106.27$ $\mathrm{cm}^{3} \cdot \mathrm{mol}^{-1}$, calculated as the ratio between the molar mass and the density), $X_{1}$ is the Flory Huggins polymer-solvent interaction parameter that for toluene is $\sim 0.391,{ }^{28}$ and $\nu_{2, \mathrm{~s}}$ is calculated using eq 5

$$
v_{2, s}=\frac{\frac{1}{\rho_{\text {polymer }}}}{\frac{Q_{\mathrm{m}}}{\rho_{\text {solvent }}}+\frac{1}{\rho_{\text {polymer }}}}
$$

where $\rho_{\text {polymer }}$ and $\rho_{\text {solvent }}$ are the densities of the polymer and of the solvent, respectively. The swelling factor $Q_{m}$ is calculated using the following equation

$$
Q_{\mathrm{m}}=\frac{w_{\mathrm{s}}-w_{\mathrm{d}}}{w_{\mathrm{s}}}
$$

where $w_{s}$ and $w_{\mathrm{d}}$ are the swelled mass and the swelled dry mass at the equilibrium, respectively.

The test was performed at room temperature, keeping the rectangular specimens in toluene for 2 days. The complete dissolution of the thermosets was tested in the dimethylformamide (DMF) solution of $5 \mathrm{wt} \%$ of $1-4$ dithiothreitol for $24 \mathrm{~h}$ at $50{ }^{\circ} \mathrm{C}$. All of the solvent tests were conducted on small rectangular $10 \times 10 \times 2 \mathrm{~mm}^{3}$ specimens.

\section{RESULTS AND DISCUSSION}

The copolymerization reactions of the EVOs with the DTBA hardener were studied using three different initiators, i.e., imidazole (IM), 1-methylimidazole (1-MI), and N,N-dimethylbenzylamine (DMBA) (respectively, D, E, and F in Figure 1 ). The correlation of EVO's structure and their epoxy index vs thermoset properties is part of the aim of this study. As references, the epoxidized linseed oil (ELO) and the epoxidized soybean oil (ESO) were chosen because (i) these two EVOs have epoxy indexes in the middle point of the variation range of the newly synthesized and herein studied EVOs and (ii) because both ELO and ESO are commercially available, produced, and applied at the industrial scale. Moreover, numerous studies highlighted the reactivity of these two epoxidized monomers with anhydride or acid hardeners. ${ }^{29-34}$ The evolution of the thermoset's properties in the function of the EVO structure will be analyzed considering EVO's monomers with comparable structures as the ELO and ESO references (i.e., same natures of fatty acids) but will also be compared with epoxidized castor oil (ECO) to gain knowledge on the influence of the additional hydroxyl groups present in its structure on the thermoset material performances. For all aforementioned systems, we will discuss further the properties of the final thermoset materials as a function of their epoxy content.

Copolymerization Reaction Investigation by DSC and FTIR Studies. The copolymerization reactions of the EVOs with DTBA catalyzed by IM, 1-MI, and DMBA were investigated by DSC to analyze if the EVOs show comparable reactivity in function of the initiators, as the reference system ELO/DTBA/initiator.

The dynamic DSC analyses were performed without the initiator and also with three selected initiators: IM, 1-MI, and DMBA. The DSC results are displayed in Figure S1. First, we can highlight the very good reactivity between the EVO's monomer and the DTBA hardener. Indeed, all of the studied systems are characterized by high values of reaction enthalpies, even in the systems without initiators. Moreover, the uncatalyzed systems react with a higher reaction enthalpy than the systems with initiators (Table S2). This result could be attributed to the occurrence of concomitant main and secondary reactions, such as homopolymerization or etherification. In comparison, the initiators introduce a selectivity through the EVOs/DTBA copolymerization; therefore, it decreases the starting temperature and the enthalpy of the reaction is given mainly by the main reaction. According to these results, it appears clearly that IM, as demonstrated for ELO, ${ }^{19,20}$ allows higher conversions during copolymerization, 
together with a lower interval of the reaction for all of the EVOs/DTBA systems.

According to our previous results, ${ }^{19,20}$ the ELO/DTBA reaction in the presence of $1-\mathrm{MI}$ is characterized by a low reaction enthalpy and low onset temperatures, $T_{\text {on }}$, compared with those of the system initiated by IM (Table S2). Thus, 1MI activates the conversion of epoxides at lower temperatures but with a lower selectivity of the copolymerization reaction. Thereafter, when DMBA is used as the catalyst, an efficient activation of the copolymerization reaction is produced, with the high reaction enthalpy for epoxidized karanja oil (EKRNO), ECO, epoxidized rapeseed oil (ERPO), and epoxidized hemp oil (EHO) monomers, but the reaction temperature range is shifted to higher temperatures. This result, combined with the low copolymerization conversions obtained for the ELO reference, made us to discard also this initiator from the subsequent studies.

An exception to this trend is the ECO/DTBA formulation, which has a particular behavior, that is, a higher reactivity and a lower reaction temperature was obtained without using a catalyst. This result can be attributed to the effect of the hydroxyl groups present in the ECO's structure.

In conclusion, IM was selected as the optimal initiator for the EVOs/DTBA copolymerization studies.

The copolymerization and reactivity of the ELO/DTBA/IM system were then considered as the reference and, therefore, the reactivities of the new EVOs were compared with this formulation. In Table 2 and Figure S2, the comparison of the DSC thermograms of EVOs/DTBA/IM copolymerization reactions is reported. The DSC results display that the enthalpy of the copolymerization reaction increases with the EVO's epoxy content (Table 2 and Figure 2). The EVOs with

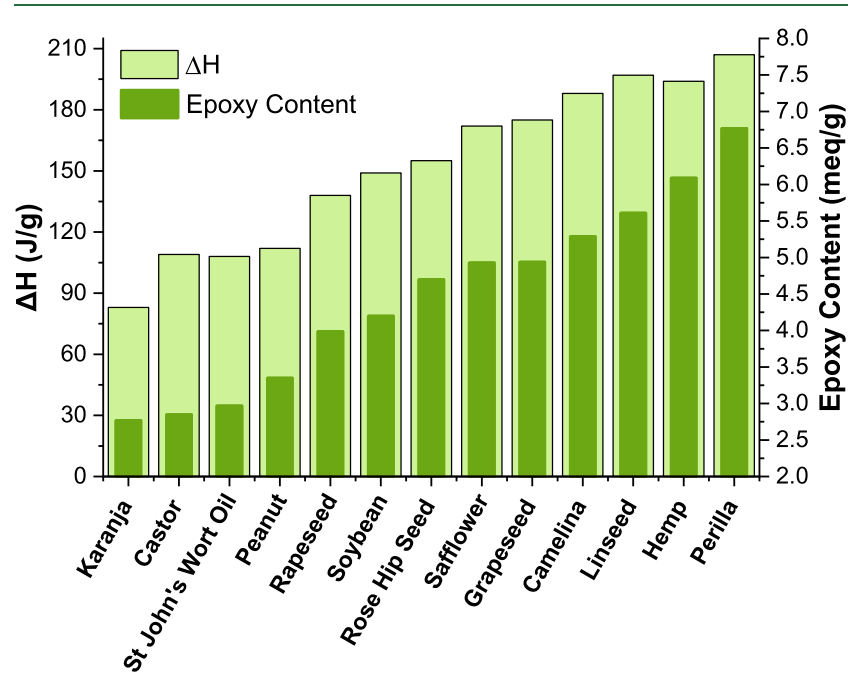

Figure 2. Correlation of the EVOs/DTBA/IM copolymerization enthalpy and monomers' epoxy content.

low epoxy content, such as EKRNO or epoxidized St John's wort oil (ESJWO) with 2.77 and 2.97 mequiv. ${ }^{-1}$, exhibit lower copolymerization enthalpy (84 and $108 \mathrm{~J} \cdot \mathrm{g}^{-1}$ ) compared with epoxidized perilla oil (EPLO) characterized by the highest epoxy content and, thus, the highest enthalpy of copolymerization $\left(207 \mathrm{~J} \cdot \mathrm{g}^{-1}\right)$. With the increase of the EVO's epoxy content, we can notice an increase of the temperature interval of the reaction. As we can observe in Figure S2, the copolymerization exothermic peaks have comparable $T_{\text {endset }}$ but the $T_{\text {onset }}$ values appear at lower temperatures for the EVOs with higher epoxy content.

FTIR analyses of the samples before and after curing provide the direct experimental indication of the involved copolymerization reactions, following the characteristic epoxy and carboxylic acid reactive groups. As example, Figure 3 illustrates

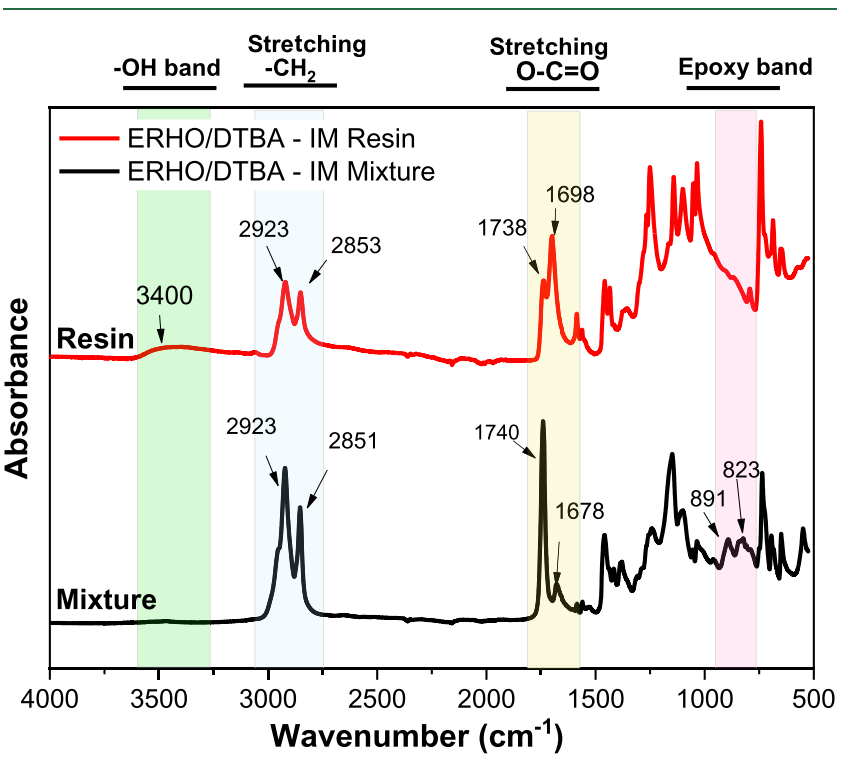

Figure 3. FTIR spectra comparison of the ERHO/DTBA/IM uncured mixture and of the thermoset resin.

the structural evolution and the comparison between the FTIR spectra of the epoxidized rosehip seed oil (ERHO)/DTBA/IM reactive mixture and the thermoset resin. In the FTIR spectrum of the uncured mixture, we can notice the oxirane characteristic bands at 823 and $891 \mathrm{~cm}^{-1}$ that completely disappear in the spectrum of the cured material. In the thermoset's spectrum, we can notice that the increased $-\mathrm{OH}$ peak at $1678 \mathrm{~cm}^{-1}$ in the mixture disappears after curing, being $\sim 3400 \mathrm{~cm}^{-1}$. Moreover, the formation of the new ester peak, with the stretching of the carbonyl $\left(\mathrm{C}=\mathrm{O}_{\text {ester }}\right)$, appears in the resin spectrum at $1738 \mathrm{~cm}^{-1}$.

In compliance with the DSC analysis, the ECO system gave different FTIR results for the epoxy monomers and for the thermosets, due to the presence of alcohol groups in their structure. The FTIR spectrum of the ECO monomer (Figure 4) shows the presence of the typical bands of $-\mathrm{OH},-\mathrm{CH}_{2}$, and $\mathrm{O}-\mathrm{C}=\mathrm{O}$ at, respectively, $\sim 3400,2925,2855$, and 1737 $\mathrm{cm}^{-1}$, while the absorption band of the characteristic epoxide ring vibration appears at $844 \mathrm{~cm}^{-1}$. In the spectrum of the thermoset material, a new ester peak appears but we should notice the presence of few residual epoxy peaks at $865 \mathrm{~cm}^{-1}$. The presence of these residual groups can be explained by the fact that the DTBA can also react with the $-\mathrm{OH}$ functions from the ECO structure. Therefore, the DTBA is in defect regarding the main copolymerization stoichiometry.

The copolymerization conversions for the EVO thermosets were calculated by applying eq 1 . The results are summarized in Figure 5 and Table S4. EKRNO and ESJWO-based thermosets display the highest conversion (97\%) in the series of EVOs, except for the reference, $\mathrm{ELO}^{19,21}$ (99\%). Conversions superior to $90 \%$ were also obtained for epoxidized peanut oil (EPO) (95\%), ERPO (94\%), ECO (92\%), and epoxidized grapeseed oil (EGRO) (92\%). 


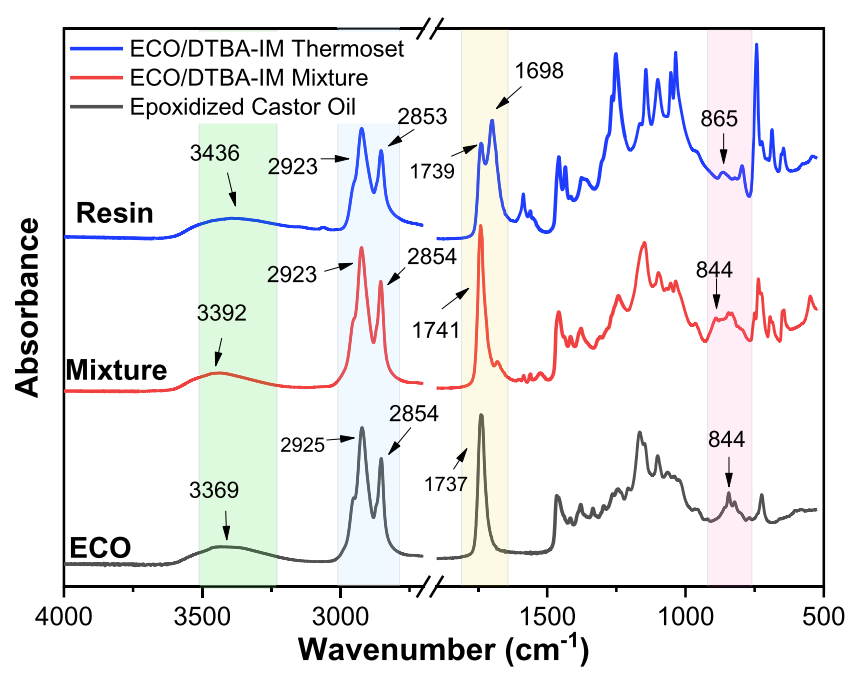

Figure 4. FTIR results and comparison between the ECO monomer, the ECO/DTBA/IM mixture, and the final thermoset.

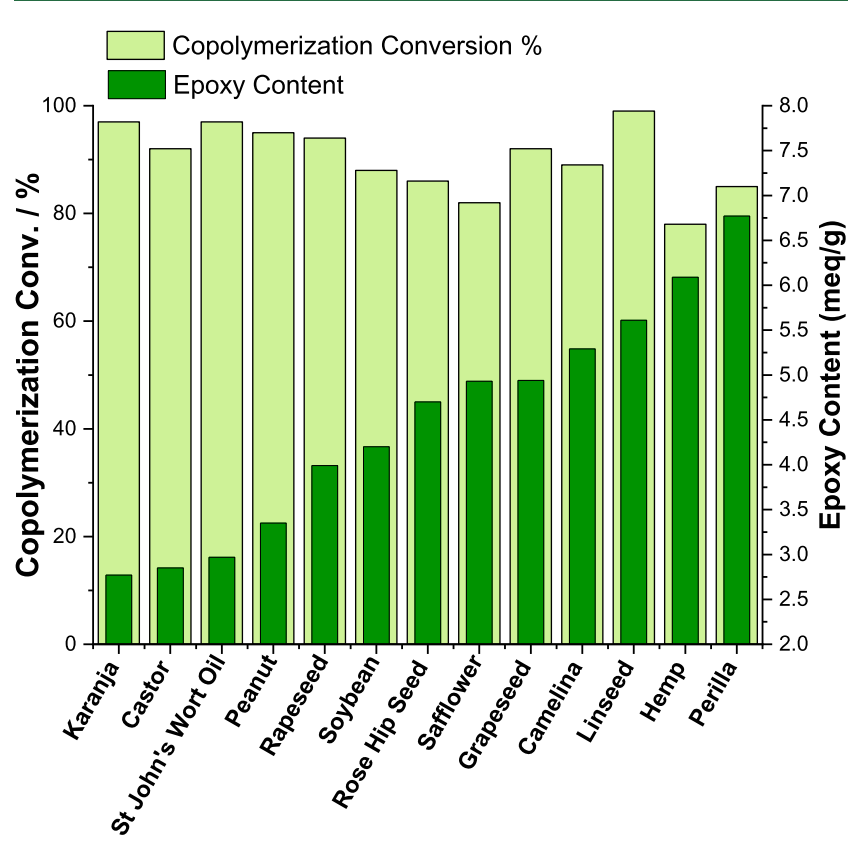

Figure 5. Conversion of epoxy functions for the EVO thermosets correlated with the epoxy content.

EHO shows the lowest conversion compared to EVOs having similar structures. This result has to be correlated to the larger copolymerization reaction range and predicts a lower $T_{\mathrm{g}}$ value for this thermoset. The same trend was observed for epoxidized safflower oil (ESFO)-based thermoset.

Thermomechanical Properties of EVOs/DTBA/IM Thermosets. Figure 6 shows the results of DMA analysis, i.e., the temperature dependence of loss factor $(\tan \delta$ ) (Figure $6 \mathrm{~A}$ ) and of the storage modulus $\left(E^{\prime}\right)$ (Figure 6B) for the prepared thermosets. The values of DMA data are listed in Table 3. We can notice that all of the thermosets reveal a single $\tan \delta$ peak, as in the case of $T_{\mathrm{g}}$ measurements performed by DSC, highlighting the existence of homogeneous networks. As shown in Figure 6 and Table 3, we can observe an increase of the $\tan \delta$ values with the epoxy content in the copolymer's formulation. The $\tan \delta$ value varies from $33{ }^{\circ} \mathrm{C}$, for thermosets made with EVOs with low epoxy content, to $111^{\circ} \mathrm{C}$, for EPLO having the highest epoxy content $\left(6.77\right.$ mequiv $\left.\cdot \mathrm{g}^{-1}\right)$. To compare the viscoelastic results, both the evolution of $T_{\mathrm{g}}$, determined by DSC, and that of $\tan \delta$, measured by DMA, are plotted in Figure 7, showing that the data fit with a linear trend in function of the epoxide content.

In line with these results, the evolution of storage modulus with the temperature (Figure 6B) shows high $E^{\prime}$ values for the thermosets based on EVOs having high epoxy content. The calculation made using eq 3 allows one to evaluate the crosslink density of the prepared network. Once again, the results show that increasing the epoxy content leads to an increase in the thermoset cross-link density from 0.025 (EKRNO thermoset) to $3.0 \mathrm{mmol} \cdot \mathrm{cm}^{-3}$ (EPLO thermoset). We can notice that EPLO and the reference ELO thermosets are the resins with the highest cross-linking (Table 3), while thermosets with low epoxy content exhibit the lowest crosslinking density (Figure 11).

ECO-based thermoset material shows the $\alpha$ transition at 52 ${ }^{\circ} \mathrm{C}$ and the lowest modulus in the rubbery plateau. Besides its low epoxy content, this low value of $E^{\prime}$ could be correlated with the lower reactivity with the DTBA hardener. However, considering all aforementioned outcomes obtained for this system, the main hypothesis explaining these results is the presence of the hydroxyl groups in the ECO structure and its possible reaction with DTBA leading to imbalanced stoichiometry and, thus, to incomplete epoxy/DTBA curing reactions. Moreover, secondary reactions as esterification or etherification implying the $-\mathrm{OH}$ reactions with $-\mathrm{COOH}$ or with epoxy functions could occur the copolymerization reaction, according to Hernandez et al. ${ }^{35}$ A large range of $T_{\mathrm{g}}$ values was obtained in the series of prepared thermosets, from $17{ }^{\circ} \mathrm{C}$ for EKRNO-based thermoset to $91{ }^{\circ} \mathrm{C}$ for EPLO-based resin. Figure $7 \mathrm{~A}$ shows the evolution of this transition in function of the EVO epoxy content. These results are also presented in function of $\tan \delta$ values correlated with the epoxy content, as shown in Figure $7 \mathrm{~B}$.

Likewise, in the study of copolymerization reactivity, we can highlight that the increase of the epoxy content leads to an increase of the corresponding $T_{\mathrm{g}}$ values of thermosets. In the case of ECO thermosets, higher values of $T_{\mathrm{g}}$ were measured, $\sim 27{ }^{\circ} \mathrm{C}$, again probably due to the presence of alcohol moieties in the structures participating in the cross-linking reactions, or in the hydrogen bonding, compared to the EVOs with the similar epoxy content as ESJWO, $T_{\mathrm{g}} \sim 17{ }^{\circ} \mathrm{C}$. These results are consistent with those reported by Gerbase et al. ${ }^{36}$ in which the lower $T_{\mathrm{g}}$ of ESO thermosets compared with that of ELO-based resins were correlated with the ESO lower epoxy index.

The low values of $T_{\mathrm{g}}$ obtained for EVOs with low epoxy content are also in agreement with the polymers derived from EVOs with the low iodine content, as presented in the literature for ECO cured with citric acid. ${ }^{34}$ Generally, the aliphatic and flexible structures usually lead to low $T_{\mathrm{g}}$ values, compared with aromatic thermosets. For instance, Williams et al. ${ }^{7}$ prepared DGEBA/DCAs epoxy vitrimers with $T_{\mathrm{g}}$ values ranging from 50 to $70{ }^{\circ} \mathrm{C}$. Wang et al. ${ }^{37}$ reported the synthesis of ESO/methylhexahydrophthalic anhydride (MHHPA) resins with $T_{\mathrm{g}} \sim 40{ }^{\circ} \mathrm{C}$, while an increase of mechanical properties was obtained by adding DGEBA in the blends. Kodam et al. ${ }^{4}$ investigated the epoxidized karanja oil cured with citric and tartaric acid and measured higher $T_{\mathrm{g}}$ values $\left(\sim 112{ }^{\circ} \mathrm{C}\right)$ attributed to high acid functionalities of the hardeners and to the hydroxyl groups of the starting building blocks producing 
A

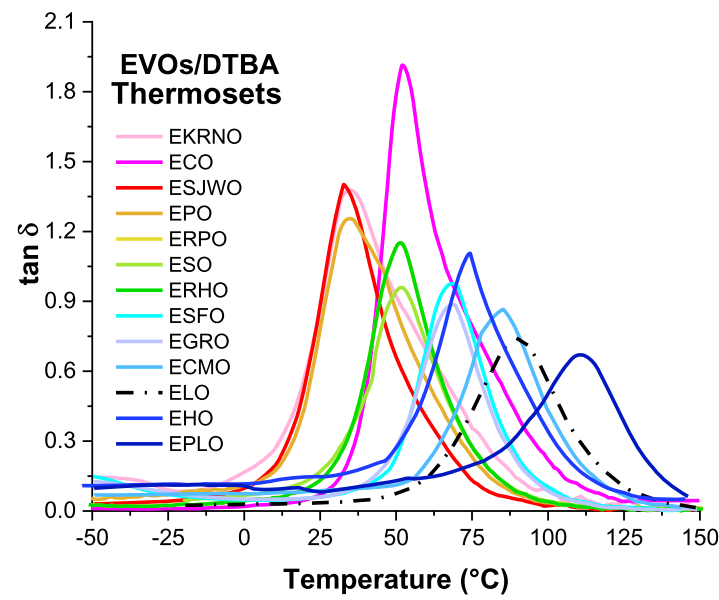

B

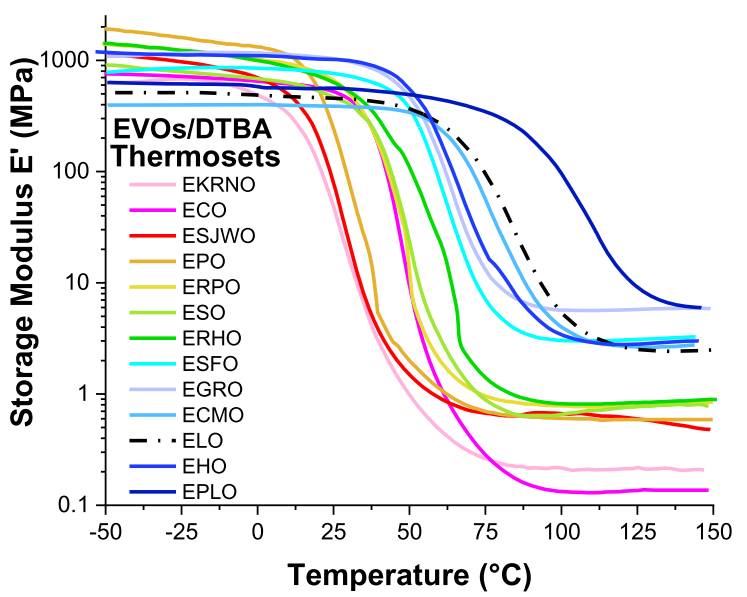

Figure 6. DMA results: $\tan \delta(\mathrm{A})$ and storage modulus $E^{\prime}(\mathrm{B})$ vs temperature for the EVOs/DTBA/IM thermosets.

\section{Table 3. Summarized DSC and DMA Data of EVOs/DTBA/IM Thermosets}

\begin{tabular}{|c|c|c|c|c|c|c|c|}
\hline EVOs/DTBA/IM thermosets & $T_{\mathrm{g}}\left({ }^{\circ} \mathrm{C}\right)$ & $\tan \delta\left({ }^{\circ} \mathrm{C}\right)$ & $(\tan \delta) \max$ & $E_{\text {glassy plateau }}^{\prime}(\mathrm{MPa})$ & $E_{\text {rubbery plateau }}^{\prime}(\mathrm{MPa})$ & cross-link density $\left(\mathrm{mmol} \cdot \mathrm{cm}^{-3}\right)$ & $\rho\left(\mathrm{g} \cdot \mathrm{cm}^{-3}\right)$ \\
\hline EKRNO & 17 & 34 & 1.40 & 630 & 0.21 & 0.025 & 1.11 \\
\hline ECO & 27 & 52 & 1.91 & 850 & 0.14 & 0.02 & 0.93 \\
\hline ESJWO & 17 & 33 & 1.40 & 1145 & 0.48 & 0.072 & 1.06 \\
\hline EPO & 24 & 35 & 1.36 & 1900 & 0.59 & 0.07 & 1.33 \\
\hline ERPO & 40 & 51 & 1.11 & 1400 & 0.82 & 0.09 & 1.35 \\
\hline ESO & 45 & 52 & 0.96 & 900 & 0.82 & 0.08 & 0.95 \\
\hline ERHO & 53 & 60 & 0.86 & 1430 & 0.92 & 0.11 & 1.13 \\
\hline ESFO & 48 & 68 & 0.99 & 780 & 3.13 & 0.31 & 1.12 \\
\hline EGRO & 50 & 67 & 0.90 & 1090 & 5.84 & 0.58 & 1.11 \\
\hline ECMO & 64 & 85 & 0.86 & 400 & 2.78 & 0.77 & 0.98 \\
\hline ELO & 76 & 91 & 0.75 & 500 & 2.63 & 1.25 & 1.02 \\
\hline EHO & 62 & 76 & 1.11 & 1200 & 3.03 & 0.75 & 1.15 \\
\hline EPLO & 91 & 111 & 0.68 & 630 & 5.98 & 3.00 & 0.98 \\
\hline
\end{tabular}
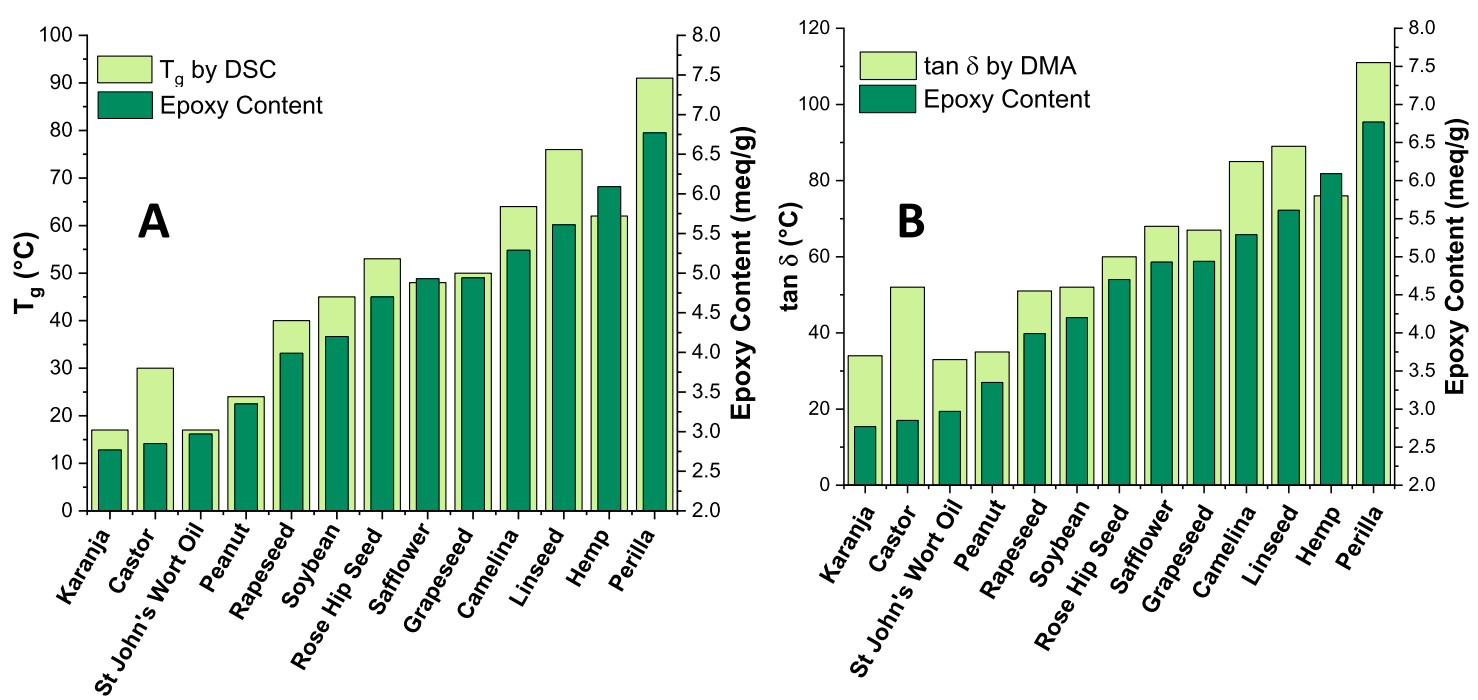

Figure 7. Glass transition evaluated by dynamic DSC analysis (A) and $\tan \delta$ (B) measured by DMA analysis in function of the EVO's epoxy content.

higher cross-linking density. Similarly, high $T_{\mathrm{g}}$ values were obtained by curing ELO with methylhexahydrophthalic anhydride (MHHPA) obtaining thermosets with $\alpha$-transition of $134{ }^{\circ} \mathrm{C}$, whereas ELO cured with 3,3,4,4-benzophenonetetracarboxylic dianhydride (BTDA) lead to cross-linked resins with $\tan \delta$ around $245{ }^{\circ} \mathrm{C} .{ }^{32}$ In contrast, Zeng et al. ${ }^{29}$ obtained materials with $T_{\mathrm{g}}$ values from -16 to $-21{ }^{\circ} \mathrm{C}$ for $\mathrm{ESO} /$ aliphatic diacid cross-linkers.

Therefore, the $T_{\mathrm{g}}$ values are strongly dependent not only on the EVO structure but also on the nature and structure of the 

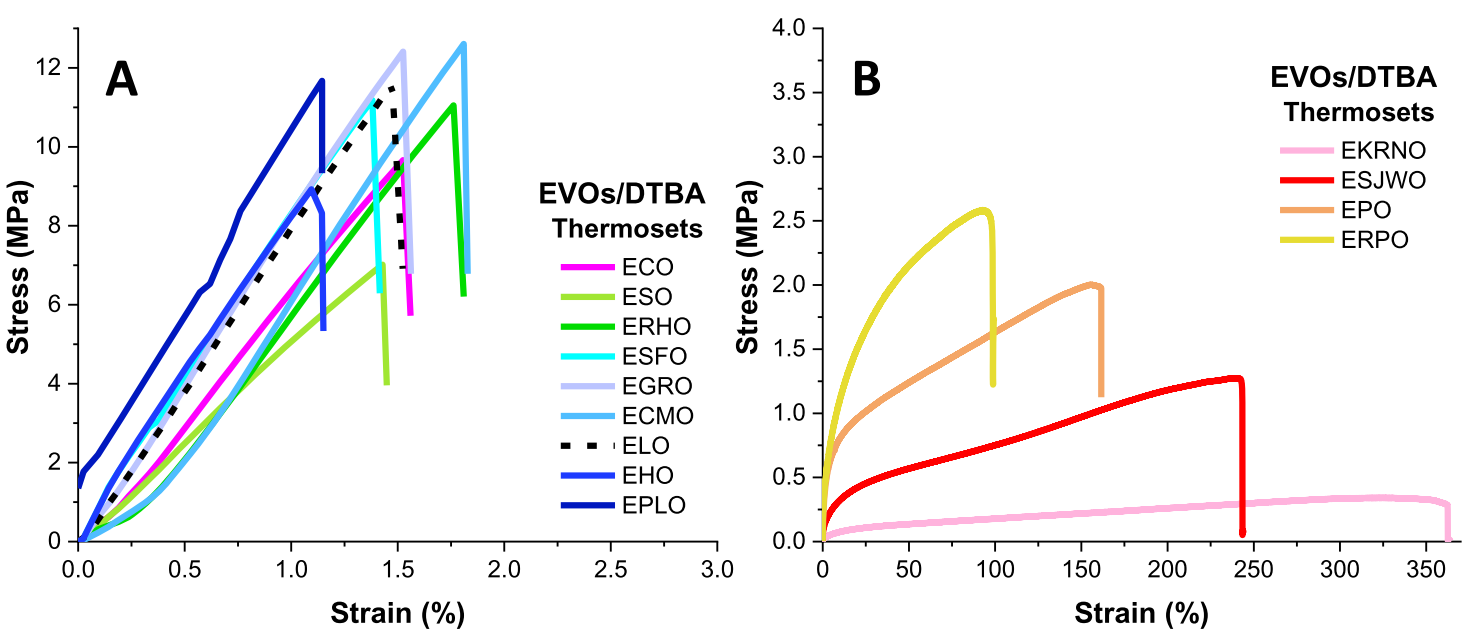

Figure 8. Stress-strain curves of EVOs/DTBA-based thermosets (A) stiff and (B) ductile EVO-based thermosets.

hardener. A proper selection of the hardener contributes to the achievement of high performances even if the EVOs have aliphatic structures. Here, without changing the hardener structure, we succeeded to reach a high $T_{\mathrm{g}}$ value, of $91{ }^{\circ} \mathrm{C}$, by copolymerization of epoxidized perilla oil with DTBA.

The static mechanical properties of the samples were studied by tensile testing. Figure 8 shows the stress-strain curves of EVOs/DTBA thermosets, and Table 4 summarizes the main

Table 4. Tensile Results (Averaged) for the EVOs/DTBA Thermosetting Resins

$\begin{array}{lccr}\begin{array}{c}\text { EVOs/DTBA/IM } \\ \text { thermosets }\end{array} & \begin{array}{c}\text { Young modulus } \\ (\mathrm{MPa})\end{array} & \begin{array}{c}\text { stress at break, } \\ \sigma(\mathrm{MPa})\end{array} & \begin{array}{r}\text { elongation at } \\ \text { break, } \varepsilon(\%)\end{array} \\ \text { EKRNO } & 0.73 \pm 0.08 & 0.39 \pm 0.07 & 347.2 \pm 54.7 \\ \text { ECO } & 731.6 \pm 59.2 & 7.96 \pm 1.85 & 1.21 \pm 0.36 \\ \text { ESJWO } & 2.42 \pm 0.77 & 1.21 \pm 0.09 & 247.5 \pm 11.0 \\ \text { EPO } & 21.1 \pm 8.2 & 2.03 \pm 0.21 & 167.4 \pm 25.5 \\ \text { ERPO } & 476.2 \pm 74.9 & 2.73 \pm 0.42 & 117.4 \pm 0.82 \\ \text { ESO } & 752.2 \pm 20.2 & 10.3 \pm 3.03 & 1.70 \pm 0.15 \\ \text { ERHO } & 771.3 \pm 39.2 & 10.1 \pm 4.38 & 2.04 \pm 0.55 \\ \text { ESFO } & 804.0 \pm 32.54 & 11.5 \pm 1.47 & 1.42 \pm 0.20 \\ \text { EGRO } & 825.5 \pm 31.93 & 12.7 \pm 2.75 & 1.62 \pm 0.38 \\ \text { ECMO } & 830.6 \pm 37.3 & 12.1 \pm 1.95 & 2.04 \pm 0.29 \\ \text { ELO } & 811.0 \pm 36.05 & 10.9 \pm 1.10 & 1.36 \pm 0.13 \\ \text { EHO } & 822.1 \pm 96.6 & 9.82 \pm 0.67 & 1.21 \pm 0.18 \\ \text { EPLO } & 815.3 \pm 10.1 & 11.5 \pm 4.55 & 1.23 \pm 0.68\end{array}$

tensile results. From Figure 8, we can notice that two kinds of stress-strain results were obtained, separating the synthesized thermosets into hard and brittle (Figure 8A) and soft and plastic (Figure 8B). The EVOs with, in general, low epoxy content and, therefore, low cross-linked networks behave as soft and flexible, which is well consistent with the trend of their $T_{\mathrm{g}}$. From Figure 8B, we can notice that these EVOs, based on ERPO, EPO, ESJWO, and EKRNO, have low to very low Young's modulus and tensile strength and the stress at break values ranging from 2.73 to $0.39 \mathrm{MPa}$. These resins exhibited high elongation at the break, from 117 to $347 \%$. These results are in good agreement with the results reported by Imbernon et al. $^{38}$ for elastomers synthetized from epoxidized natural rubber and dithiodibutyric acid cross-linker.

In contrast, the thermosets obtained from EVOs with an epoxy content $>4.20$ mequiv. $^{-1}$ reveal higher tensile strength and Young's modulus values. These thermosets have strength at the break at around $10-12 \mathrm{MPa}$, while the elongation values are very low, $<2 \%$, characteristics for hard and brittle thermosets.

A peculiar trend shows the ECO-based thermoset that has a tensile behavior close to that of thermosets based on EVOs with high epoxy content, despite the fact that ECO is from the category of EVOs with low content, $\sim 2.85$ mequiv $\cdot \mathrm{g}^{-1}$. This result can be again explained by the contribution of the hydroxyl functions present in its structure to increase the network chemical connectivity, confirmed by its $T_{\mathrm{g}}$ and $\tan \delta$ values, superior to those of thermosets from EVOs with a comparable epoxy content.

All of the EVO thermosets exhibit better mechanical properties than some other EVO-based polymer networks, which were cured by carboxylic acids ${ }^{4,29,33,39}$ or the EVOs/ disulfide cross-linker ${ }^{40}$ that showed lower $\sigma$ and $\varepsilon$. These results open the window for industrial applications.

The literature shows that starting from vegetable oils, a large diversity of derivatives can be synthesized with very good properties. For example, Webster and Pan developed highperformance thermosets by preparing epoxidized sucrose esters of fatty acids (ESEFAs) (soybean, linseed, safflower) crosslinked with 4-methylhexahydrophthalic anhydride (MHHPA) using two ratios of epoxy/anhydride of 1:0.5 and 1:0.75. ${ }^{41}$ The modulus and tensile strength of ESS (epoxidized sucrose soyate) were higher than those of the ESO control thermoset by factors of 7.6 and 2, respectively, while the properties of the ESL (epoxidized sucrose linseedate) thermoset were even higher with a modulus 21 times superior and a tensile strength 4.5 times greater than that of the ESO thermoset. The same authors reported increased properties, ${ }^{42}$ replacing ESO crosslinked with dodecenylsuccinic anhydride (DDSA), dipentaerythritol (DPE), tripentaerythritol (TPE), or sucrose esters of soybean oil, synthesized using transesterification from soybean methyl ester and the corresponding polyols. The increased values in $T_{\mathrm{g}}$ and moduli were correlated to the presence of the available hydroxyl groups, which can facilitate the formation of polyester networks through the rapid reaction between the hydroxyl and anhydride.

Wang et al. ${ }^{43}$ reported very good properties of epoxy polymer derivatives of soybean oil, synthesized by amidation of soybean oil with three amino alcohols (1-amino-2-propanol, 3amino- 1-propanol, and 1,3-diamino-2-propanol), followed by (meth)acrylation, then by the free radical polymerization of 


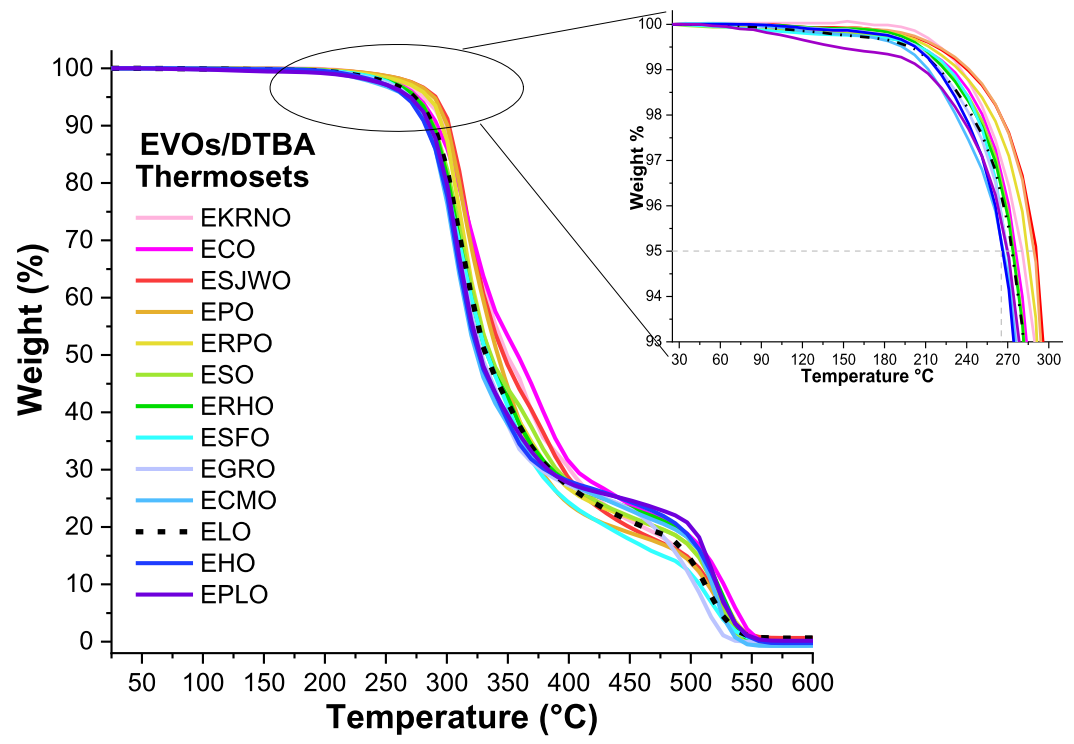

Figure 9. TGA thermograms during heating the EVO thermosets at $10^{\circ} \mathrm{C} \cdot \mathrm{min}^{-1}$ in air; zoom in the region $25-320{ }^{\circ} \mathrm{C}$.

unsaturated fatty side chains, and finally, the epoxidation of unsaturations. The authors obtained an increased tensile strength and modulus replacing ESO/4-methyl-1,2-cyclohexanedicarboxylic anhydride with these soybean oil epoxy polymers, obtaining values from 1.4 MPa (control, ESO-based thermoset) to $37 \mathrm{MPa}$ for the tensile strength and from 2.4 $\mathrm{MPa}$ (ESO-based thermoset) to $561 \mathrm{MPa}$ Young's modulus.

Zhou et al. ${ }^{44}$ observed that the mechanical strength, Young's modulus, and toughness improved with the increase of the epoxy group density for sunflower oil-based epoxy copolymers EP(SFMA-co-VM) synthesized by the sunflower oil-based monomer (SFMA) and vanillin-derived methacrylate (VM).

The thermal stability profiles of the obtained thermosets are displayed in Figures 9 and S3 and Tables 5 and S5. All of the

Table 5. $T_{5 \%}$ and $T_{s}$ Values Determined by TGA Analysis

$\begin{array}{lcc}\text { EVOs/DTBA/IM thermosets } & T_{5 \%}\left({ }^{\circ} \mathrm{C}\right) & T_{s}\left({ }^{\circ} \mathrm{C}\right) \\ \text { EKRNO } & 285 & 171 \\ \text { ECO } & 275 & 169 \\ \text { ESJWO } & 290 & 170 \\ \text { EPO } & 285 & 164 \\ \text { ERPO } & 285 & 172 \\ \text { ESO } & 271 & 167 \\ \text { ERHO } & 275 & 165 \\ \text { ESFO } & 271 & 162 \\ \text { EGRO } & 265 & 161 \\ \text { ECMO } & 265 & 162 \\ \text { ELO } & 275 & 167 \\ \text { EHO } & 265 & 164 \\ \text { EPLO } & 265 & 159\end{array}$

EVO thermosets show similar degradation behavior in air, with good thermal stability, in the same range of ELO thermoset reference. The $T_{5 \%}$ values are ranging from $265^{\circ} \mathrm{C}$ for EPLO thermosets to $290^{\circ} \mathrm{C}$ for ESJWO. Resins of EVOs with low epoxy content exhibit higher thermal stability, whereas increasing the epoxy content decreases the thermal stability. The reason for this behavior could be connected to the amount of DTBA in the network, and consequently to the S-S bonds in the resins. Ma et al. ${ }^{45}$ reported that the presence of $S-S$ bonds provokes a decrease of thermoset's thermal stability due to their lower dissociation energy compared with that of $\mathrm{C}-\mathrm{C}$ bonds. Moreover, higher the oxirane ring content, more ester and hydroxyl groups are created through the curing. These functions can also promote the thermal scissions of the networks.

The thermal stability of the EVO thermosets are similar to those of epoxy resins containing disulfide dynamic bonds, ${ }^{46}$ but lower if we compare it with ELO/adipic acid or glutaric anhydride systems ${ }^{33}$ or with ESO/dicarboxylic acid (DCA) thermosets with different carbon chain-lengths. ${ }^{29}$ Figure S3 displays the derivative thermogravimetric analysis (DTG) curves highlighting the main degradation steps.

The statistic heat-resistant index temperature values $\left(T_{s}\right)$ (Table 5) have a trend similar to the $T_{5 \%}$ values. For the thermosets based on EVOs with low epoxy content, ESJWO and EKRNO thermosets, the $T_{s}$ values are higher compared to the reported values for epoxidized sucrose soyate cured with citric or malic acids ${ }^{47}\left(148-154^{\circ} \mathrm{C}\right)$. Supanchaiyamat et al. ${ }^{48}$ correlated the first thermal degradation stage with the breaking of the ester linkages, while the second one with the cleavage of the products formed during the first degradative step.

Solvent Stabilities and Chemical Degradability Behavior. To evaluate the solvent stability of the EVO thermosets, weighed and measured samples were immersed for $72 \mathrm{~h}$ in methanol, ethanol, acetone, toluene, $1 \mathrm{~N} \mathrm{NaOH}$, and 1 $\mathrm{N} \mathrm{HCl}$. Three thermosets were selected to analyze how the low or high epoxy content impact on solvent stability: ESJWO, ECO, and EPLO thermosets. Their behavior after immersion in the mentioned solvents was compared (Figure 10A-C). After $72 \mathrm{~h}$, these resins were generally stable in all solvents and no changes were observed in $1 \mathrm{~N} \mathrm{HCl}$ solution.

ECO resin (Figure 10C) shows similar behavior as ESJWO and EPLO resins. Gerbase et al. ${ }^{36}$ reported higher chemical resistance for ESO cured with cyclic anhydrides in polar organic solvents (acetone, ethanol) than in toluene where an interaction of polymer-solvent by network's swelling was observed. For DGEBA cured with 4-aminophenyl disulfide, Ruiz de Luzuriaga et al. ${ }^{49}$ showed that the materials were not affected after immersion at room temperature for $72 \mathrm{~h}$ in $1 \mathrm{~N}$ $\mathrm{NaOH}$ and $\mathrm{HCl}, \mathrm{THF}$, toluene, acetone, and ethanol. 
A

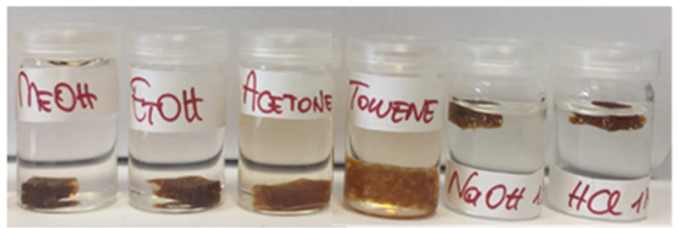

B

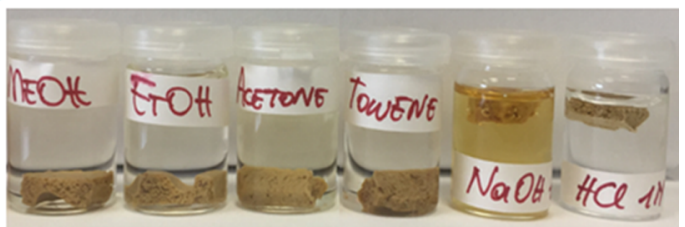

c

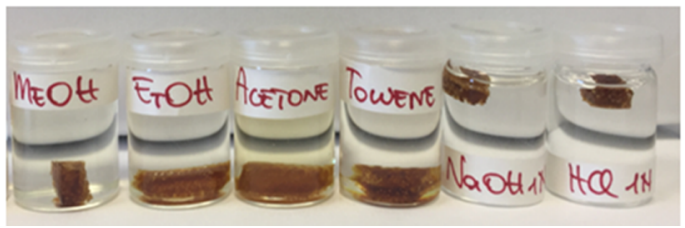

Figure 10. Solvent stability study for selected thermosets: ESJWO (A), EPLO (B), and ECO (C) after $72 \mathrm{~h}$ in methanol, ethanol, acetone, toluene, $1 \mathrm{~N} \mathrm{NaOH}$, and $1 \mathrm{~N} \mathrm{HCl}$.

We already proved the ELO/DTBA thermoset disintegration in $1 \mathrm{~N} \mathrm{NaOH}$ at $80{ }^{\circ} \mathrm{C}$ or at room temperature after 3 days. $^{20}$ This behavior could be attributed to a synergic effect: the disulfide metathesis ${ }^{50,51}$ and also the cleavage of the ester bonds. As example, $\mathrm{Ma}$ et $\mathrm{al}^{45}$ reported that the isosorbidederived epoxy cross-linked with 4-aminophenyl disulfide exhibits a complete degradation after $3 \mathrm{~h}$ in alkaline solution, attributing this result to $S-S$ cleavage and to the water-soluble nature of isosorbide.

From Figure 10B, we can observe that EPLO resin presents a faster autodegradation in alkaline solution compared to ESJWO thermoset due to its higher epoxy content and, consequently, due to a higher content on ester connections formed during cross-linking.

Regarding the swelling tests, toluene was selected as a solvent for this test for $24 \mathrm{~h}$ at room temperature. The results are traced and plotted in Figure 11. Vitrimers are known for

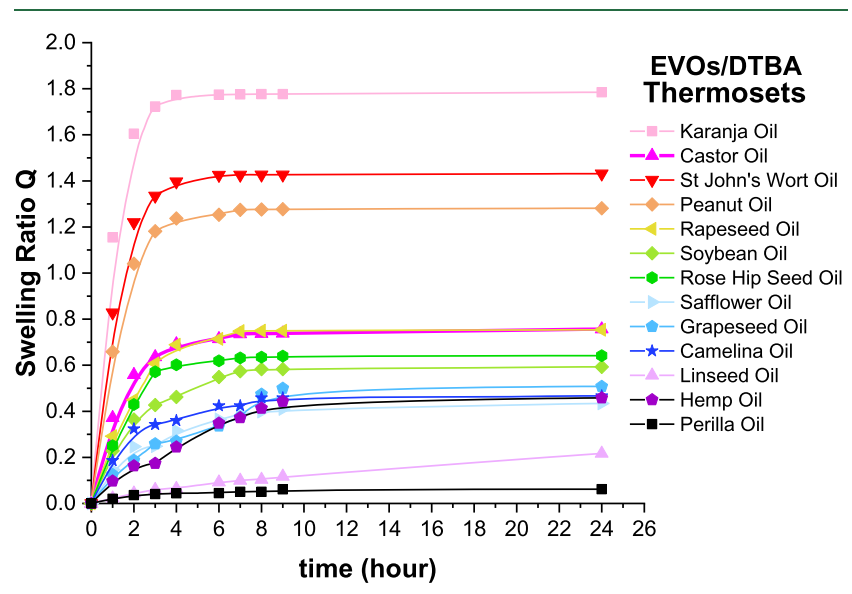

Figure 11. Swelling tests performed in toluene for $24 \mathrm{~h}$.

"swell, but do not dissolve, in good solvents" as reported by Leibler et al., 52 and the swelling equilibrium of the thermosets in given solvents is an indicator of the cross-linking. ${ }^{53}$ The degree of swelling is known to be dependent on the cross-link density of thermoset networks. The higher the cross-link density, the lower is the degree of swelling: EPLO has the lowest swelling ability. EKRNO and ESJWO thermosets show the highest swelling, confirming the lowest network cross-link density. The swelling ratio of the EVOs is lower than that of acrylated epoxidized soybean oil (AESBO)/maleic anhydride thermosets $^{48}$ or of other bio-based thermosets. ${ }^{54}$

The swelling test results were used further to determine the cross-link density of the networks, as described by FloryRehner theory. ${ }^{26,27}$ Applying this method, the network density values were evaluated by measuring their swelling in toluene at room temperature for $48 \mathrm{~h}$. Previously, we determined by DMA data (Figure 12) that the cross-linking significantly

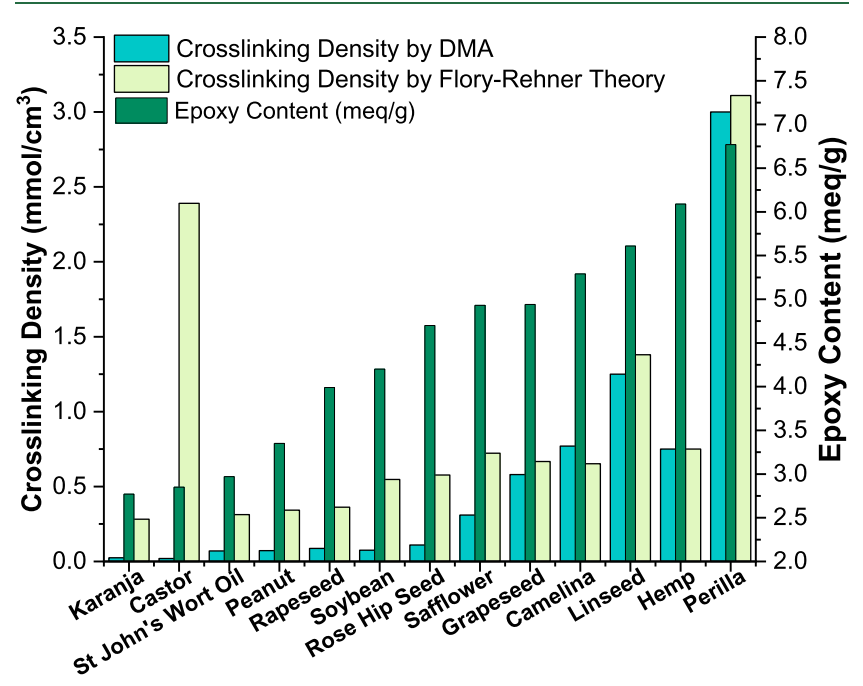

Figure 12. Cross-linking density is determined by the rubber elasticity and by Flory-Rehner theories, in function of the EVO epoxy content.

increased with the epoxy content, as confirmed by the rubberyelasticity theory. The results are reported and compared in Table S6. The corroborated data by the two analyses demonstrate the same trend but with higher values for the results extracted by the swelling tests. For the thermosets based on EVOs with high epoxy content, the results are close, especially for EPLO and EHO materials. A contradictory result has been observed for ECO thermoset with one of the highest cross-linking densities measured by Flory-Rehner theory.

Finally, the reduction of the disulfide bonds to thiols was performed using a DMF solution with 5 wt \% of dithiothreitol, according to Zhou et al., ${ }^{46}$ at $50{ }^{\circ} \mathrm{C}$ for $24 \mathrm{~h}$. Similar results were obtained by Ruiz de Luzuriaga et al., ${ }^{49}$ who showed a complete dissolution of DGEBA/4-aminophenyl disulfide 
thermosets in 2-mercaptoethanol in DMF solution immersed at room temperature for $24 \mathrm{~h}$.

Figure 13 displays this reduction for ERHO thermoset resin. The thiol groups present in the solvent solution react with the

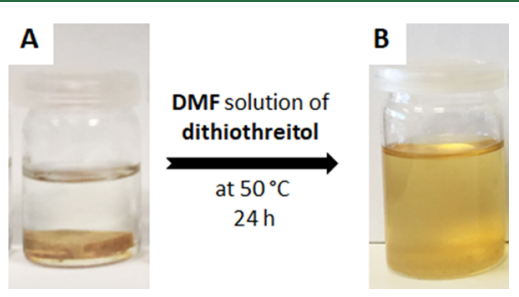

Figure 13. ERHO thermoset (A) before and (B) after immersion in DMF solution with dithiothreitol.

cross-linked dynamic disulfide networks, producing a complete debonding of the $\mathrm{S}-\mathrm{S}$ connections. This complete thermosets' dissolution opens important windows to chemical recyclability for thermoset materials.

Bio-Based Content. Finally, the bio-based content of the EVOs/DTBA/IM thermosets was evaluated as "the amount of bio-based carbon in the material or product as a percent of the weight (mass) of the total organic carbon in the product". 55

All of the starting EVO monomers were obtained from renewable materials, as seeds of vegetables, while the hardener and the initiator are not bio-based products. Considering ELO thermoset formulation, the carbon content in the starting materials is: in ELO $~ 70.5 \%$, in DTBA $\sim 47 \%$, and in IM $53 \%$, respectively. Therefore, for the ratio $\mathrm{EVO} /$ hardener $=1 /$ 1: $2.5 \mathrm{~g}$ of ELO reacts with $2.149 \mathrm{~g}$ of DTBA and $0.046 \mathrm{~g}$ of IM, consequently the bio-based content according to Pan et al. $^{41}$ is

$$
\begin{aligned}
& \text { bio-based content } \% \\
& =\frac{(2.5 \mathrm{~g} \times 70.5 \%)}{(2.5 \mathrm{~g} \times 70.5 \%)+(2.149 \mathrm{~g} \times 47 \%)+(0.046 \mathrm{~g} \times 53 \%)} \\
& =63.0 \% \\
& \text { carbon bio-based content } \% \\
& =\frac{\text { bio-based carbon }}{\text { bio-based carbon }+ \text { petro-based carbon }} \\
& \text { carbon bio-based content } \% \\
& =\frac{57 \times 0.532}{57 \times 0.532+14 \times 0.458+3 \times 0.01} \\
& =82.5 \%
\end{aligned}
$$

The bio-based content determined for all of the thermosets (Table S7) is in the range from $79 \%$ for EKRNO to $58 \%$ for EPLO, while the carbon bio-content varies from $91 \%$ for EKRNO to $80 \%$ for EPLO. Increasing the epoxy content, the bio-based content and the carbon bio-content decrease due to a higher amount of needed hardener in the formulation.

\section{CONCLUSIONS}

A series of novel bio-based epoxy thermosets were developed using a broad range of epoxidized vegetable oils. These EVO monomers were copolymerized with a disulfide aromatic dicarboxylic acid (DCA) and, therefore, the reactivity of these systems was compared. The new EVOs allow one to obtain materials with a wide range of thermomechanical properties, thanks to their variation in chemical structures and concomitant reactivities with the acid hardener. By an appropriate choice of epoxy content in the EVO structure, the final properties could be predicted. Indeed, the copolymerization of EVOs with DTBA produced materials, exhibiting either high $T_{\mathrm{g}} \sim 91{ }^{\circ} \mathrm{C}, \tan \delta$ values of $111^{\circ} \mathrm{C}$, and cross-link density of $3 \mathrm{mmol} \cdot \mathrm{cm}^{-3}$ as in the case of EPLO or low $T_{\mathrm{g}}$ values $\left(\sim 17{ }^{\circ} \mathrm{C}\right)$ as in the case of EKRNO-based thermoset, characterized by a low epoxy content. The large range of tensile properties, from soft and plastic (for thermosets based on EVOs with an epoxy content <4 mequiv $\cdot \mathrm{g}^{-1}$ ) to hard and rigid could be used to develop novel sustainable thermosetting materials with modulated properties and, thus, of interest for very different application fields. The obtained materials, with a high bio-based carbon content $>80 \%$ can find applications in protective coatings, structural matrix, and composites.

The chemical recyclability results are opening windows for a future work that will be dedicated to the investigation of $3 R$ ability (recyclability, repairability, and reshapability) of these new EVO thermosets.

\section{ASSOCIATED CONTENT}

\section{Supporting Information}

The Supporting Information is available free of charge at https://pubs.acs.org/doi/10.1021/acs.biomac.0c01059.

Characteristics of the selected reagents; thermocuring studies of the reaction of the EVOs/DTBA with and without initiators by DSC analysis; curing and postcuring conditions for the EVOs/DTBA/IM; thermoset material characterization by TGA, DMA, ATRFTIR, and bio-based content and carbon bio-content \% for EVOs/DTBA/IM (PDF)

\section{AUTHOR INFORMATION}

\section{Corresponding Author}

Alice Mija - Universite Côte d'Azur, Institut de Chimie de Nice, UMR CNRS 7272, 06108 Nice, France; ○ orcid.org/00000001-5208-5956; Email: Alice.Mija@univ-cotedazur.fr

\section{Authors}

Chiara Di Mauro - Université Côte d'Azur, Institut de Chimie de Nice, UMR CNRS 7272, 06108 Nice, France

Samuel Malburet - SPECIFIC POLYMERS, 34160 Castries, France; (1) orcid.org/0000-0002-4224-9520

Aratz Genua - Basque Research and Technology Alliance (BRTA), CIDETEC, Donostia-San Sebastián 20014, Spain

Alain Graillot - SPECIFIC POLYMERS, 34160 Castries, France

Complete contact information is available at:

https://pubs.acs.org/10.1021/acs.biomac.0c01059

\section{Notes}

The authors declare no competing financial interest.

\section{ACKNOWLEDGMENTS}

This work was supported by the ECOXY project. ECOXY is a project funded by the European Commission. This project has received funding from the Bio-Based Industries Joint Undertaking under the European Union's Horizon 2020 research and innovation program (Grant agreement no. 744311). 


\section{ABBREVIATIONS}

VO, vegetable oil; EVO, epoxidized vegetable oil; ELO, epoxidized linseed oil; EKRNO, epoxidized karanja oil; ECO, epoxidized castor oil; ESJWO, epoxidized St John's wort oil; EPO, epoxidized peanut oil; ERPO, epoxidized rapeseed oil; ESO, epoxidized soybean oil; ERHO, epoxidized rosehip seed oil; ESFO, epoxidized safflower oil; EGRO, epoxidized grapeseed oil; ECMO, epoxidized camelina oil; EHO, epoxidized hemp oil; EPLO, epoxidized perilla oil; IM, imidazole; 1-MI, 1-methyl imidazole; DMBA, N,N'-dimethyl benzamine; DTBA, 2,2'-dithiodibenzoic acid; DGEBA, diglycidyl ether of bisphenol A; DCA, dicarboxylic acids; FTIR, Fourier transform infrared spectroscopy; DSC, differential scanning calorimetry; DMA, dynamic mechanical analysis; TGA, thermogravimetric analysis; DTG, derivative thermogravimetric analysis; DMF, dimethylformamide; AESBO, acrylated epoxidized soybean oil; MHHPA, methylhexahydrophthalic anhydride

\section{REFERENCES}

(1) Parada Hernandez, N. L.; Bonon, A. J.; Bahú, J. O.; Barbosa, M. I. R.; Wolf Maciel, M. R.; Filho, R. M. Epoxy monomers obtained from castor oil using a toxicity-free catalytic system. J. Mol. Catal. A: Chem. 2017, 426, 550-556.

(2) Lathi, P. S.; Mattiasson, B. Green approach for the preparation of biodegradable lubricant base stock from epoxidized vegetable oil. Appl. Catal., B 2007, 69, 207-212.

(3) Wu, X.; Zhang, X.; Yang, S.; Chen, H.; Wang, D. The study of epoxidized rapeseed oil used as a potential biodegradable lubricant. J. Am. Oil Chem. Soc. 2000, 77, 561-563.

(4) Kadam, A.; Pawar, M.; Yemul, O.; Thamke, V.; Kodam, K. Biodegradable biobased epoxy resin from karanja oil. Polymer 2015, $72,82-92$.

(5) Eissen, M.; Metzger, J. O.; Schmidt, E.; Schneidewind, U. 10 Years after Rio-Concepts on the Contribution of Chemistry to a Sustainable Development. Angew. Chem., Int. Ed. 2002, 41, 414-436.

(6) Capelot, M.; Unterlass, M. M.; Tournilhac, F.; Leibler, L. Catalytic Control of the Vitrimer Glass Transition. ACS Macro Lett. 2012, 1, 789-792.

(7) Altuna, F. I.; Casado, U.; dell'Erba, I. E.; Luna, L.; Hoppe, C. E.; Williams, R. J. J. Epoxy vitrimers incorporating physical crosslinks produced by self-association of alkyl chains. Polym. Chem. 2020, 11, 1337-1347.

(8) García, F.; Smulders, M. M. J. Dynamic covalent polymers. J. Polym. Sci., Part A: Polym. Chem. 2016, 54, 3551-3577.

(9) Biermann, U.; Friedt, W.; Lang, S.; Lühs, W.; Machmüller, G.; Metzger, J. O.; Rüsch gen. Klaas, M.; Schäfer, H. J.; Schneider, M. P. New Syntheses with Oils and Fats as Renewable Raw Materials for the Chemical Industry. Angew. Chem., Int. Ed. 2000, 39, 2206-2224.

(10) Hammond, E. W. VEGETABLE OILS I Types and Properties. In Encyclopedia of Food Sciences and Nutrition, 2nd ed.; Caballero, B., Ed.; Academic Press: Oxford, 2003; pp 5899-5904.

(11) Xia, Y.; Larock, R. C. Vegetable oil-based polymeric materials: synthesis, properties, and applications. Green Chem. 2010, 12, 18931909.

(12) Kim, J. R.; Sharma, S. The development and comparison of biothermoset plastics from epoxidized plant oils. Ind. Crops Prod. 2012, $36,485-499$.

(13) Hong, J.; Radojčić, D.; Ionescu, M.; Petrović, Z. S.; Eastwood, E. Advanced materials from corn: isosorbide-based epoxy resins. Polym. Chem. 2014, 5, 5360-5368.

(14) Noè, C.; Malburet, S.; Bouvet-Marchand, A.; Graillot, A.; Loubat, C.; Sangermano, M. Cationic photopolymerization of biorenewable epoxidized monomers. Prog. Org. Coat. 2019, 133, 131138.
(15) Ma, S.; Liu, X.; Jiang, Y.; Tang, Z.; Zhang, C.; Zhu, J. Bio-based epoxy resin from itaconic acid and its thermosets cured with anhydride and comonomers. Green Chem. 2013, 15, 245-254.

(16) Czub, P. Synthesis of high-molecular-weight epoxy resins from modified natural oils and Bisphenol A or Bisphenol A-based epoxy resins. Polym. Adv. Technol. 2009, 20, 194.

(17) Sinadinović-Fišer, S.; Janković, M.; Petrović, Z. S. Kinetics of in situ epoxidation of soybean oil in bulk catalyzed by ion exchange resin. J. Am. Oil Chem. Soc. 2001, 78, 725-731.

(18) Danov, S. M.; Kazantsev, O. A.; Esipovich, A. L.; Belousov, A. S.; Rogozhin, A. E.; Kanakov, E. A. Recent advances in the field of selective epoxidation of vegetable oils and their derivatives: a review and perspective. Catal. Sci. Technol. 2017, 7, 3659-3675.

(19) Tran, T.-N.; Di Mauro, C.; Graillot, A.; Mija, A. Chemical Reactivity and the Influence of Initiators on the Epoxidized Vegetable Oil/Dicarboxylic Acid System. Macromolecules 2020, 53, 2526-2538.

(20) Di Mauro, C.; Tran, T.-N.; Graillot, A.; Mija, A. Enhancing the Recyclability of a Vegetable Oil-Based Epoxy Thermoset through Initiator Influence. ACS Sustainable Chem. Eng. 2020, 8, 7690-7700.

(21) Di Mauro, C.; Genua, A.; Mija, A. Building thermally and chemically reversible covalent bonds in vegetable oils based epoxy thermosets. Influence of epoxy-hardener ratio to promote recyclability Mater. Adv. 2020, DOI: 10.1039/D0MA00370K.

(22) Bowman, C. N.; Kloxin, C. J. Covalent Adaptable Networks: Reversible Bond Structures Incorporated in Polymer Networks. Angew. Chem., Int. Ed. 2012, 51, 4272-4274.

(23) Azcune, I.; Odriozola, I. Aromatic disulfide crosslinks in polymer systems: Self-healing, reprocessability, recyclability and more. Eur. Polym. J. 2016, 84, 147-160.

(24) Chen, J.-H.; Hu, D.-D.; Li, Y.-D.; Meng, F.; Zhu, J.; Zeng, J.-B. Castor oil derived poly(urethane urea) networks with reprocessibility and enhanced mechanical properties. Polymer 2018, 143, 79-86.

(25) Matějka, L.; Lövy, J.; Pokorný, S.; Bouchal, K.; Dušek, K. Curing epoxy resins with anhydrides. Model reactions and reaction mechanism. J. Polym. Sci., Polym. Chem. Ed. 1983, 21, 2873.

(26) Flory, P. J. Principles of Polymer Chemistry; Cornell University Press, 1953.

(27) Treloar, L. R. G. The Physics of Rubber Elasticity; Oxford University Press: USA, 1975.

(28) Onn, M.; Nor, H.; Khairuddin, W. A. Development of Solid Rocket Propellant based on Isophorone Diisocyanate - Hydroxyl Terminated Natural Rubber Binder. J. Teknol. 2014, 69, 53-58.

(29) Zeng, R.-T.; Wu, Y.; Li, Y.-D.; Wang, M.; Zeng, J.-B. Curing behavior of epoxidized soybean oil with biobased dicarboxylic acids. Polym. Test. 2017, 57, 281-287.

(30) Falco, G.; Sbirrazzuoli, N.; Mija, A. Biomass derived epoxy systems: From reactivity to final properties. Mater. Today Commun. 2019, 21, No. 100683.

(31) Gobin, M.; Loulergue, P.; Audic, J.-L.; Lemiègre, L. Synthesis and characterisation of bio-based polyester materials from vegetable oil and short to long chain dicarboxylic acids. Ind. Crops Prod. 2015, 70, 213-220.

(32) Pin, J. M.; Sbirrazzuoli, N.; Mija, A. From epoxidized linseed oil to bioresin: An overall approach of epoxy/anhydride cross-linking. ChemSusChem 2015, 8, 1232.

(33) Ding, C.; Tian, G.; Matharu, A. Adipic acid - glutaric anhydride - epoxidised linseed oil biobased thermosets with tunable properties. Mater. Today Commun. 2016, 7, 51-58.

(34) Pin, J.-M.; Guigo, N.; Vincent, L.; Sbirrazzuoli, N.; Mija, A. Copolymerization as a Strategy to Combine Epoxidized Linseed Oil and Furfuryl Alcohol: The Design of a Fully Bio-Based Thermoset. ChemSusChem 2015, 8, 4149-4161.

(35) Parada Hernandez, N. L.; Bahú, J. O.; Schiavon, M. I. R. B.; Bonon, A. J.; Benites, C. I.; Jardini, A. L.; Maciel Filho, R.; Wolf Maciel, M. R. Epoxidized castor oil - citric acid) copolyester as a candidate polymer for biomedical applications. J. Polym. Res. 2019, 26, No. 149. 
(36) Gerbase, A. E.; Petzhold, C. L.; Costa, A. P. O. Dynamic mechanical and thermal behavior of epoxy resins based on soybean oil. J. Am. Oil Chem. Soc. 2002, 79, 797-802.

(37) Wang, R.; Schuman, T. P. Vegetable oil-derived epoxy monomers and polymer blends: A comparative study with review. eXPRESS Polym. Lett. 2013, 7, 272-292.

(38) Imbernon, L.; Oikonomou, E. K.; Norvez, S.; Leibler, L. Chemically crosslinked yet reprocessable epoxidized natural rubber via thermo-activated disulfide rearrangements. Polym. Chem. 2015, 6, 4271-4278.

(39) Supanchaiyamat, N.; Shuttleworth, P. S.; Hunt, A. J.; Clark, J. H.; Matharu, A. S. Thermosetting resin based on epoxidised linseed oil and bio-derived crosslinker. Green Chem. 2012, 14, 1759.

(40) Liu, Y.-Y.; He, J.; Li, Y.-D.; Zhao, X.-L.; Zeng, J.-B. Biobased, reprocessable and weldable epoxy vitrimers from epoxidized soybean oil. Ind. Crops Prod. 2020, 153, No. 112576.

(41) Pan, X.; Sengupta, P.; Webster, D. C. High Biobased Content Epoxy-Anhydride Thermosets from Epoxidized Sucrose Esters of Fatty Acids. Biomacromolecules 2011, 12, 2416-2428.

(42) Pan, X.; Webster, D. C. Impact of structure and functionality of core polyol in highly functional biobased epoxy resins. Macromol. Rapid Commun. 2011, 32, 1324-1330.

(43) Wang, Z.; Yuan, L.; Ganewatta, M. S.; Lamm, M. E.; Rahman, M. A.; Wang, J.; Liu, S.; Tang, C. Plant Oil-Derived Epoxy Polymers toward Sustainable Biobased Thermosets. Macromol. Rapid Commun. 2017, 38, No. 1700009.

(44) Zhou, J.; Xu, K.; Xie, M.; Wu, H.; Hua, Z.; Wang, Z. Two Strategies to precisely tune the mechanical properties of plant oilderived epoxy resins. Composites, Part B 2019, 173, No. 106885.

(45) Ma, Z.; Wang, Y.; Zhu, J.; Yu, J.; Hu, Z. Bio-based epoxy vitrimers: Reprocessibility, controllable shape memory, and degradability. J. Polym. Sci., Part A: Polym. Chem. 2017, 55, 1790-1799.

(46) Zhou, F.; Guo, Z.; Wang, W.; Lei, X.; Zhang, B.; Zhang, H.; Zhang, Q. Preparation of self-healing, recyclable epoxy resins and lowelectrical resistance composites based on double-disulfide bond exchange. Compos. Sci. Technol. 2018, 167, 79-85.

(47) Ma, S.; Kovash, C. S.; Webster, D. C. Effect of solvents on the curing and properties of fully bio-based thermosets for coatings. J. Coat. Technol. Res. 2017, 14, 367-375.

(48) Supanchaiyamat, N.; Hunt, A. J.; Shuttleworth, P. S.; Ding, C.; Clark, J. H.; Matharu, A. S. Bio-based thermoset composites from epoxidised linseed oil and expanded starch. RSC Adv. 2014, 4, 23304-23313.

(49) Ruiz de Luzuriaga, A.; Martin, R.; Markaide, N.; Rekondo, A.; Cabañero, G.; Rodríguez, J.; Odriozola, I. Epoxy resin with exchangeable disulfide crosslinks to obtain reprocessable, repairable and recyclable fiber-reinforced thermoset composites. Mater. Horiz. 2016, 3, 241-247.

(50) Takahashi, A.; Ohishi, T.; Goseki, R.; Otsuka, H. Degradable epoxy resins prepared from diepoxide monomer with dynamic covalent disulfide linkage. Polymer 2016, 82, 319-326.

(51) Lei, Z. Q.; Xiang, H. P.; Yuan, Y. J.; Rong, M. Z.; Zhang, M. Q. Room-Temperature Self-Healable and Remoldable Cross-linked Polymer Based on the Dynamic Exchange of Disulfide Bonds. Chem. Mater. 2014, 26, 2038-2046.

(52) Montarnal, D.; Capelot, M.; Tournilhac, F.; Leibler, L. Silicalike malleable materials from permanent organic networks. Science 2011, 334, 965.

(53) Luo, Q.; Liu, M.; Xu, Y.; Ionescu, M.; Petrović, Z. S. Thermosetting Allyl Resins Derived from Soybean Oil. Macromolecules 2011, 44, 7149-7157.

(54) Faye, I.; Decostanzi, M.; Ecochard, Y.; Caillol, S. Eugenol biobased epoxy thermosets: from cloves to applied materials. Green Chem. 2017, 19, 5236-5242.

(55) Norton, G. A.; Devlin, S. L. Determining the modern carbon content of biobased products using radiocarbon analysis. Bioresour. Technol. 2006, 97, 2084-2090. 